\title{
Chinese herbal medicine for functional dyspepsia: systematic review of systematic reviews
}

\author{
Michael H. K. Chu, Irene X. Y. Wu, Robin S. T. Ho, Charlene H. L. Wong, Anthony L. Zhang,
} Yan Zhang, Justin C. Y. Wu and Vincent C. H. Chung

\begin{abstract}
Background: Pharmacotherapy, including prokinetics and proton pump inhibitors for functional dyspepsia (FD) have limited effectiveness, and their safety has been recently questioned. Chinese herbal medicine ( $\mathrm{CHM}$ ) could be considered as an alternative. A systematic review (SR) of SRs was performed to evaluate the potential effectiveness and safety of $\mathrm{CHM}$.

Method: We conducted a comprehensive literature search for SRs with meta-analyses in eight international and Chinese databases. Pooled effect estimation from each meta-analysis was extracted. The AMSTAR instrument was used to assess the methodological quality of the included SRs.

Results: A total of 14 SRs of mediocre quality assessing various $\mathrm{CHMs}$, alone or in combination with conventional pharmacotherapy, were included. Meta-analyses showed that $\mathrm{CHM}$ was more effective than prokinetic agents for the alleviation of global dyspeptic symptoms. Three specific CHM formulae appeared to show superior results in the alleviation of global dyspeptic symptoms, including Si Ni San, modified Xiao Yao San and Xiang Sha Liu Jun Zi decoction. No significant difference in the occurrence of adverse events in using $\mathrm{CHM}$ or pharmacotherapy was reported.

Conclusion: $\mathrm{CHM}$ can be considered as an alternative for the treatment of FD symptoms when prokinetic agents and proton pump inhibitors are contraindicated. Future trial design should focus on measuring changes in individual dyspeptic symptoms and differentiate the effectiveness of different CHM for postprandial distress syndrome and epigastric pain syndrome. A network meta-analysis approach should be used to explore the most promising CHM formula for FD treatment in the future.
\end{abstract}

Keywords: functional dyspepsia, Chinese herbal medicine, Chinese herbs, prokinetics, proton pump inhibitors, systematic reviews

Received: 19 December 2017; revised manuscript accepted: 6 June 2018

\section{Introduction}

Functional dyspepsia (FD) is a common cause of dyspeptic symptoms in the world. ${ }^{1}$ The annual incidence of FD approximates $9-10 \%$, while $15 \%$ of patients have chronic ( $>3$ months per year) and frequent ( $>3$ episodes per week) symptoms. ${ }^{1}$ In the general population, the prevalence of FD is $11.5-14.7 \% .^{2}$ The prevalence of FD varies between $8 \%$ and $23 \%$ among Asia population. ${ }^{3}$ It can also be as high as $23.9 \%$ in Spain, $32 \%$ in the United States (US) and $38-41 \%$ in the United
Kingdom. ${ }^{1}$ FD is more prevalent in women $(24.4 \%)$ than men $(16.6 \%)$ and its occurrence was found to increase significantly with age..$^{5,6}$

Currently defined by the Rome IV criteria, FD is categorized into two types: postprandial distress syndrome (PDS) and epigastric pain syndrome (EPS). ${ }^{7}$ PDS is characterized by postprandial fullness and early satiety, while EPS is characterized by epigastric pain or burning. ${ }^{8}$ Both do not carry any structural disorders explaining the symptoms. ${ }^{8}$
Ther Adv Gastroenterol

2018, Vol. 11: 1-24

DOI: 10.1177/

1756284818785573

(c) The Author(s), 2018.

Reprints and permissions: http://www.sagepub.co.uk/ journalsPermissions.nav
Correspondence to:

Irene X. Y. Wu

Hong Kong Institute of Integrative Medicine, The Chinese University of Hong Kong, 5/F, School of Public Health Building, Prince of Wales Hospital, Shatin, New Territories, Hong Kong wuxinyinfacuhk.edu.hk

Michael H. K. Chu

Global Physician-

Leadership Stream,

MBChB program, The

Chinese University of Hong

Kong, Hong Kong

Robin S. T. Ho

Jockey Club School of

Public Health and Primary

Care, The Chinese

University of Hong Kong,

Hong Kong

Charlene H. L. Wong

Hong Kong Institute of

Integrative Medicine, The

Chinese University of

Hong Kong, Hong Kong

Department of Medicine

and Therapeutics, The

Chinese University of Hong

Kong, Hong Kong

Anthony L. Zhang

China-Australia

International Research

Centre for Chinese

Medicine School of

Health and Biomedical

Sciences, RMIT University,

Melbourne, Australia

Australian Research

Centre in Complementary

and Integrative Medicine

(ARCCIM), University

of Technology Sydney,

Sydney, Australia

Yan Zhang

Australian Research

Centre in Complementary

and Integrative Medicine

(ARCCIM), University

of Technology Sydney,

Sydney, Australia

Department of Family and Community Medicine,

Texas Tech University

Health Sciences Center,

Lubbock, TX, USA

Justin C. Y. Wu

Hong Kong Institute of

Integrative Medicine, The 
Chinese University of Hong Kong, Hong Kong Vincent C. H. Chung Hong Kong Institute of Integrative Medicine, The Chinese University of Hong Kong, Hong Kong Jockey Club School of Public Health and Primary Care, The Chinese University of Hong Kong, Hong Kong
FD does not seem to pose severe or fatal threats to patients. Nevertheless, it greatly reduces patient quality of life; ${ }^{9}$ the impact of FD on quality of life is comparable in patients with other chronic diseases such as asthma and inflammatory bowel disease. ${ }^{10,11}$ Moreover, FD incurs heavy economic burdens, attributable to medical care and diagnostic procedure costs. Although the cost data on FD is limited, statistics show that FD created an $\$ 18.4$ billion medical cost on US patients in 2009. ${ }^{12}$

There are several guideline-recommended pharmacotherapies for functional dyspepsia. ${ }^{8,9,13-15}$ In a population with high Helicobacter pylori infection rate $(>20 \%)$, FD patients should be tested and treated by antibiotics if such infection exists. In contrast, in a population with low $H$. pylori prevalence $(<20 \%)$, proton pump inhibitor (PPI) should be used for reducing gastric acid secretion in patients with epigastric pain, while prokinetic agents can be used if the symptoms include postprandial fullness or early saiety. $8,9,13-15$

However, due to the heterogeneous nature of the symptoms of $\mathrm{FD},{ }^{16}$ probably no single pharmacotherapy is able to manage the symptoms fully. ${ }^{9}$ Besides, individual symptoms may be caused by different mechanisms. As a result, the same drug may not be able to deal with the same symptom if it is originated from different mechanisms in different patients. ${ }^{9}$ In addition, despite being widely in use, prokinetic agents only demonstrate limited effectiveness. For example, acotiamide has a number needed to treat (NNT) of $16^{17}$ for symptom elimination, while the NNT figures of omeprazole and lansoprazole are $9 .{ }^{18}$ The side effects of pharmacotherapy also trigger concerns among medical practitioners. Current evidence has shown its association with extra-pyramidal reactions, cardiac arrhythmia and neurological disorders, such as Parkinsonism. ${ }^{19-22}$ There is a need for addressing the effectiveness gap between patients who are experiencing limited benefits from prokinetic agents and PPI, or patients who are contraindicated to them.

Chinese herbal medicine (CHM) is part of the routine in the treatment of FD in China and is gaining popularity in other countries. In traditional Chinese medicine (TCM), FD is equivalently termed as 'distension and fullness' (in Chinese pinyin: Pi Man), 'stomach pain' (in Chinese pinyin: Wei Wan Tong) and 'retention' (in Chinese pinyin: Ji Zhi). ${ }^{23} \mathrm{FD}$ can also be divided into various TCM syndromes, which may include, but not limited to: (a) liver-stomach disharmony syndrome; (b) dampness-heat of spleen and stomach syndrome; (3) cold and heat in complexity syndrome; and (4) spleen-stomach weakness syndrome. ${ }^{24} \mathrm{TCM}$ aims at harmonizing or improving the relationship between different organs and systems in the human body in order to relieve the symptoms of FD. Traditionally, accurate diagnosis of TCM syndrome type is considered a key for guiding the appropriate choice of individualized CHM formula, although the use of CHM without TCM diagnosis is also common.

Existing meta-analyses indicated that, in general, CHM was superior to placebo for improvement of quality of life, alleviation of global dyspeptic symptoms, and reduction of severity from a CHM perspective. ${ }^{25,26}$ When CHM is compared with pharmacotherapy, evidence from existing metaanalyses is inconsistent. Meta-analyses comparing $\mathrm{CHM}^{25,27}$ or selected specific CHM formula ${ }^{28}$ versus pharmacotherapy in general showed that CHM was more effective than pharmacotherapy for the alleviation of global dyspeptic symptoms. Meta-analyses showed CHM in general, ${ }^{29,30}$ or selected specific CHM formulae ${ }^{31-37}$ were more effective than prokinetic agents. However, another meta-analysis and one separate trial showed similar effectiveness between CHM and prokinetic agents for the alleviation of global dyspeptic symptoms and global PDS, respectively. ${ }^{38}$ For alleviation of FD individual symptoms, meta-analyses showed individual CHM formula was more effective than prokinetic agents, including alleviation of early satiety and epigastric pain, but not for postprandial fullness and epigastric burning. ${ }^{37}$

\section{Objective}

The heterogeneous results from existing systematic reviews (SRs) make it difficult to draw conclusions on the effectiveness of CHM over pharmacotherapy. There is a need to perform a SR of SRs to resolve such inconsistency. We conducted a systematic review of SRs to critically appraise and synthesize current clinical evidence on the effectiveness and safety of CHM on FD.

\section{Method}

\section{Eligibility criteria}

To be included, the SRs should meet the criteria as follows: (a) patients were diagnosed with FD 
according to Rome criteria I, II or III, or other criteria stated by the authors; (b) CHM must be included as one of the treatments, and any herbal formula, dosage, and route of administration were eligible; (c) the control group should employ conventional pharmacotherapy, including but not limited to: prokinetic agents, PPIs, H. Pylori eradication, or placebo of CHM; (d) a meta-analysis should be conducted in the SR; (e) SRs should be published in English or Chinese.

We excluded data from meta-analyses which included randomized controlled trials (RCTs) evaluating the following pharmacological therapies, of which substantial side effects had been reported: ${ }^{39-43}$ cisapride, combined therapy of PPIs and prokinetic agents, as well as combined therapy of $\mathrm{H}_{2}$ histamine receptor antagonist (H2RA) and prokinetic agents.

A comprehensive literature search was conducted by searching both international and Chinese databases from their inception till Jan 2017. International databases included the Cochrane Database of Systematic Reviews, Database of Abstracts of Reviews of Effects, MEDLINE and EMBASE. Chinese databases included Chinese Biomedical Databases, Wanfang Digital Journals, China National Knowledge Infrastructure and Taiwan Periodical Literature Databases. A specialized search filter for reviews was used in MEDLINE $^{44}$ and EMBASE. ${ }^{45}$ Detailed search strategies and related results for each database are reported in Table A1 in the appendix. No language restriction was set during the literature search.

\section{Literature selection, data extraction, and} methodological quality assessment

All the retrieved citations were screened and assessed for eligibility. Data that were extracted from the included SRs were as follows: characteristics of the SR, including first author's name, year of publication, eligibility criteria for selecting primary studies, and number of included studies and patients; details on patient characteristics, details of CHM and control interventions; effect sizes on each outcome of interest, and adverse effects; and risk of bias among RCTs included in the SRs.

The methodological quality of included SRs was appraised by the AMSTAR tool, a reliable and valid tool for methodological quality assessment of SRs. ${ }^{46,47}$ Eleven aspects were assessed using AMSTAR, with each aspect being judged as 'yes', 'no', 'can't answer' or 'not applicable', based on the information provided. The detailed operational guide for AMSTAR is provided in Table A2 in the appendix.

Literature selection, data extraction, and methodological quality assessment were performed by two researchers (MC and XY) independently. Disagreements were resolved by thorough discussions and consensus adjudication. A third reviewer (VC) would be consulted if disagreement could not be resolved, and his judgment was considered to be the final decision.

\section{Outcomes of interest}

Trials results extracted from each meta-analysis should include at least one of the following primary outcomes:

(1) Alleviation of global dyspeptic symptoms, measured by global symptom improvement scale;

(2) Alleviation of individual dyspeptic symptoms (epigastric burning, epigastric pain, gastric emptying or fullness), measured by individual symptom improvement scale.

Quality-of-life improvement and safety were considered as the secondary outcomes in this SR.

\section{Data synthesis}

The effectiveness of CHM treatments was assessed at SR level according to the Cochrane Handbook. ${ }^{48}$ No reanalyzing of the data using network meta-analysis approach was performed due to the insufficient number of trials sharing a common comparator, as well as head-to-head comparison between interventions. We extracted the pooled effect estimation from each meta-analysis. Pooled relative risk (RR) or odds ratio (OR) for dichotomous outcomes, and weighted mean difference (WMD) or standard mean difference (SMD) for continuous outcomes, accompanied by their respective $95 \%$ confidence intervals (CIs) were extracted as reported by the meta-analyses.

$I$-square $\left(I^{2}\right)$ values were also extracted for appraising heterogeneity among RCTs. The $I^{2}$ 
value of $<25 \%, 25-50 \%,>50 \%$ were regarded as an indicator for the presence of low, moderate, and high heterogeneity, respectively. ${ }^{49}$

\section{Results}

\section{Study selection}

There were a total of 601 citations retrieved from the database searches. Among them, 12 SRs were found to be eligible and were included in the SR of SRs. Two additional SRs were identified from existing SRs. ${ }^{26,28}$ Therefore, 14 SRs were inclu ded. ${ }^{25-38}$ Details on the study selection process can be found in Figure 1 .

\section{Characteristics of included systematic reviews}

The fourteen SRs were published between 2009 and 2016 and all were conducted in China. Characteristics of these SRs can be found in Table 1. Eight SRs (57.1\%) were published in English and the remaining six (42.9\%) were published in Chinese. Thirteen SRs (92.9\%) searched both international and Chinese databases. Thirteen SRs (92.9\%) included only RCTs, while the remaining one $(7.1 \%)$ included both RCTs and quasi-RCTs. Seven SRs $(50 \%)$ conducted a literature search in or after 2013, with the most recent search conducted in February 2016.

\section{Characteristics of patients}

The patients included ranged from age 14 to 87 years. Three SRs $(21.4 \%)$ included FD patients based on Rome III criteria, six (42.9\%) based on Rome II or III criteria, two (14.3\%) employed Rome I, II or III criteria. The remaining three SRs $(21.4 \%)$ followed the criteria set by the authors. The treatment duration was 2-4 weeks in eight SRs $(57.1 \%)$, but it was as long as 8 weeks in three SRs $(21.4 \%)$.

\section{Characteristics of intervention and control}

In the intervention arm, CHM was administered either alone or in combination with conventional pharmacotherapy. Eight SRs reviewed nine specific CHM formulae, namely (a) modified Xiao Yao San, (b) Liu Jun Zi decoction, (c) Xiang Sha Liu Jun Zi decoction, (d) Shu Gan Jie Yu capsule, (e) modified Chai Hu Shu Gan powder, (f) Ban Xia Xie Xin decoction, (g) Liu Wei An Xiao capsule, (h) Si Ni San and (i) Da Li Tong granule. On the other hand, the remaining six SRs summarized outcomes from different CHM formulae. Details of intervention dosage and frequency, and details of CHM evaluated are shown in Table A3 in the appendix.

In the control arm, the included trials applied either conventional pharmacotherapy or placebo of CHM. Six SRs included only trials evaluating domperidone or mosapride in the control group, while seven SRs set no restrictions on conventional pharmacotherapy used in the control group. Among these seven SRs, one of them also included CHM placebo as a control. Therefore, together with another SR accepting CHM placebo as a control, there are two SRs synthesizing evidence on CHM versus $\mathrm{CHM}$ placebo comparison.

\section{Methodological quality of included systematic}

reviews

Overall, the methodological quality of the included SRs was moderate, with 13 SRs (92.9\%) having reported the basic characteristics of the included studies. Twelve SRs $(85.7 \%)$ combined the studies' findings using appropriate methods, assessed the likelihood of publication bias, assessed and documented the scientific quality of the included studies, and their scientific quality was considered appropriately in conclusion formulation. However, only three (21.4\%) searched for grey literature and none of them provided a list of included and excluded studies. Details on the methodological quality of included SRs is shown in Table 2 .

\section{Effectiveness of Chinese herbal medicine for functional dyspepsia}

\section{Primary outcomes}

Alleviation of global dyspeptic symptoms. All the included SRs, with a total of 24 comparisons, summarized evidence of CHM on FD (Table 3). For the intervention arm, there are 13 comparisons focusing on a specific CHM formula. CHM was administered either alone (9 comparisons), or in combination with pharmacotherapy (4 comparisons). There are also 11 comparisons that included multiple types of CHM formulae as intervention, either alone (10 comparisons) or as 
Citations identified through electronic databases search $(n=601)$

EMBASE $(n=407)$

MEDLINE $(n=82)$

Cochrane Database of Systematic Reviews (CDSR; $n=43$ )

Database of Abstracts of Reviews of Effect (DARE; $n=13$ )

Wan Fang Digital Journals (Chinese; $n=32$ )

China National Knowledge Infrastructure (Chinese; $n=1$ )

Taiwan Periodical Literature Databases (Chinese $n=3$ )

Chinese Biomedical Database (Chinese; $n=20$ )

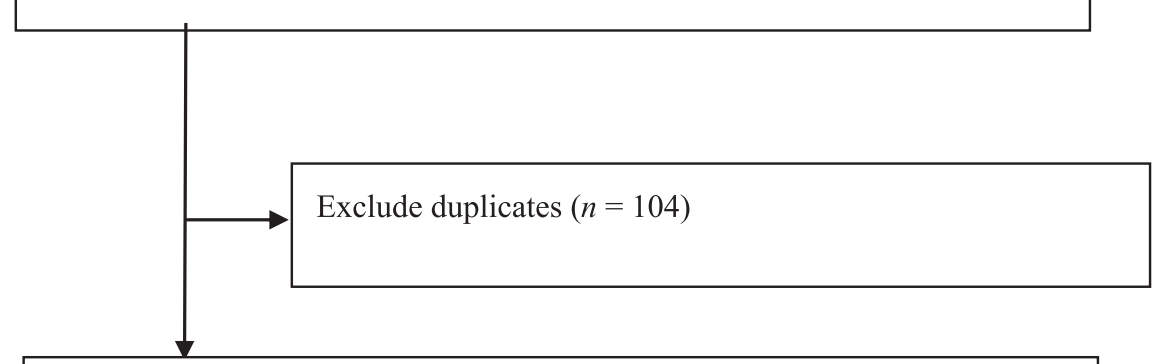

Citations for screening $(n=497)$

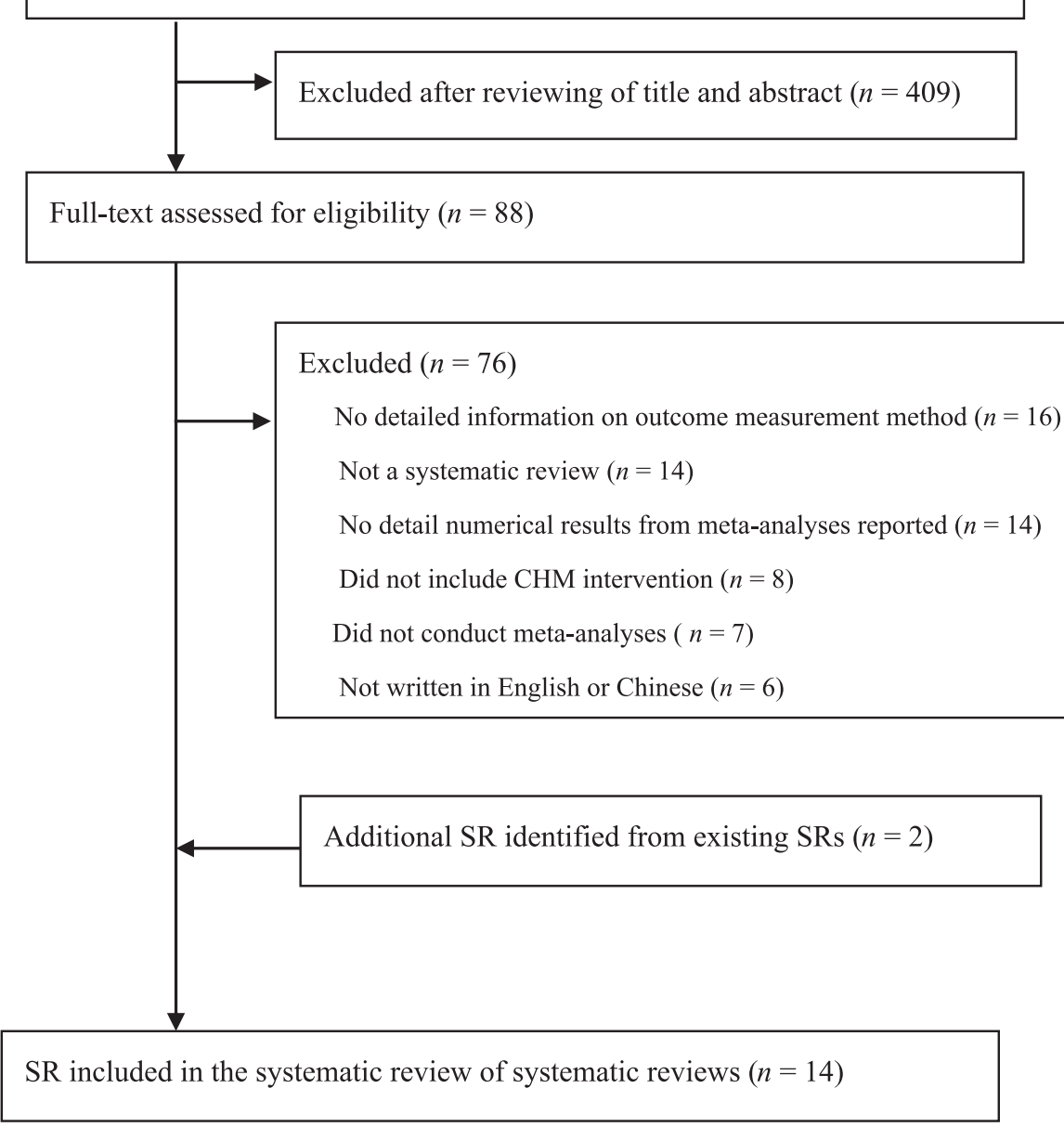

Figure1. Flowchart of literature search for systematic reviews on Chinese herbal medicine for functional dyspepsia. 


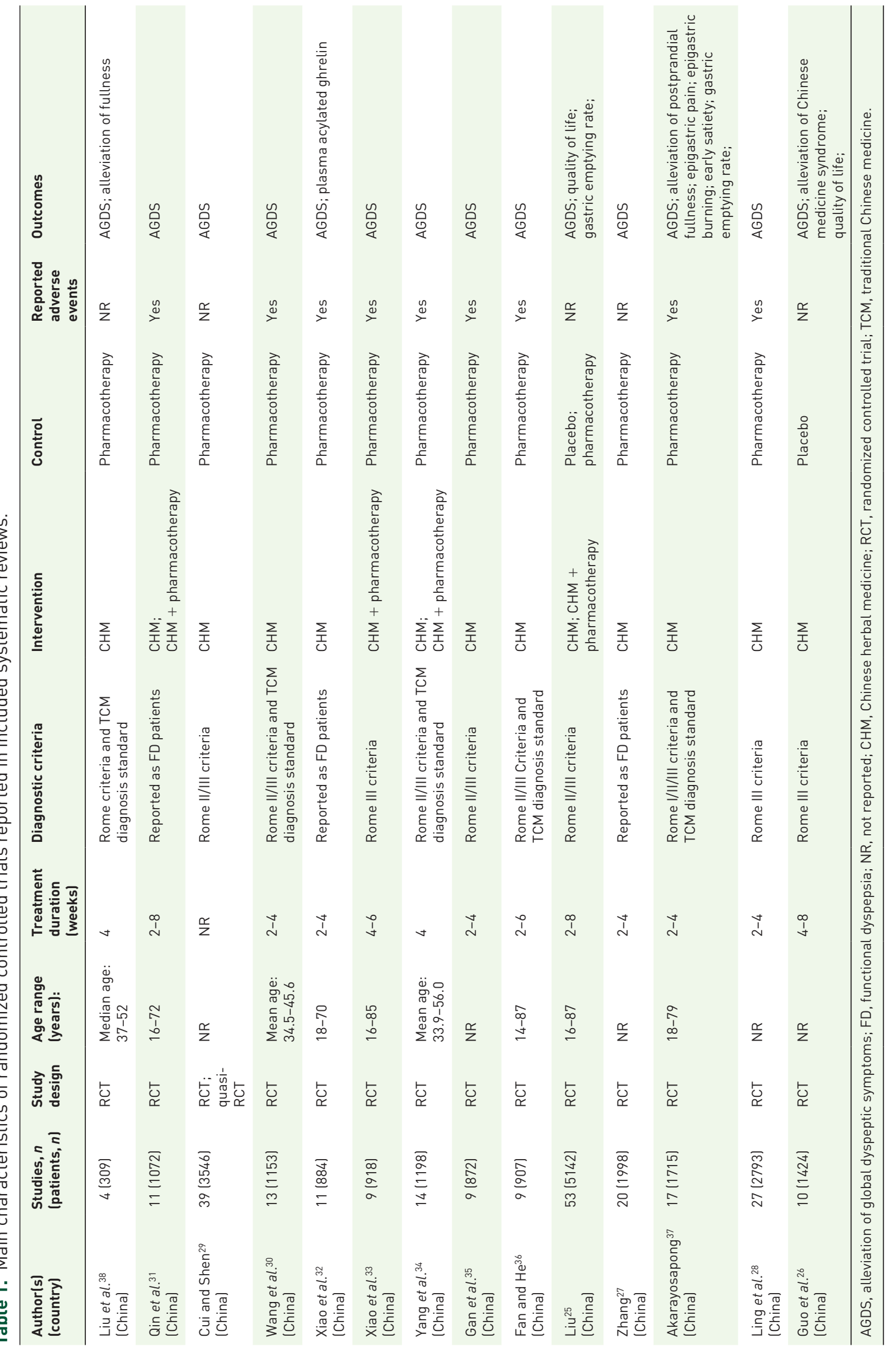




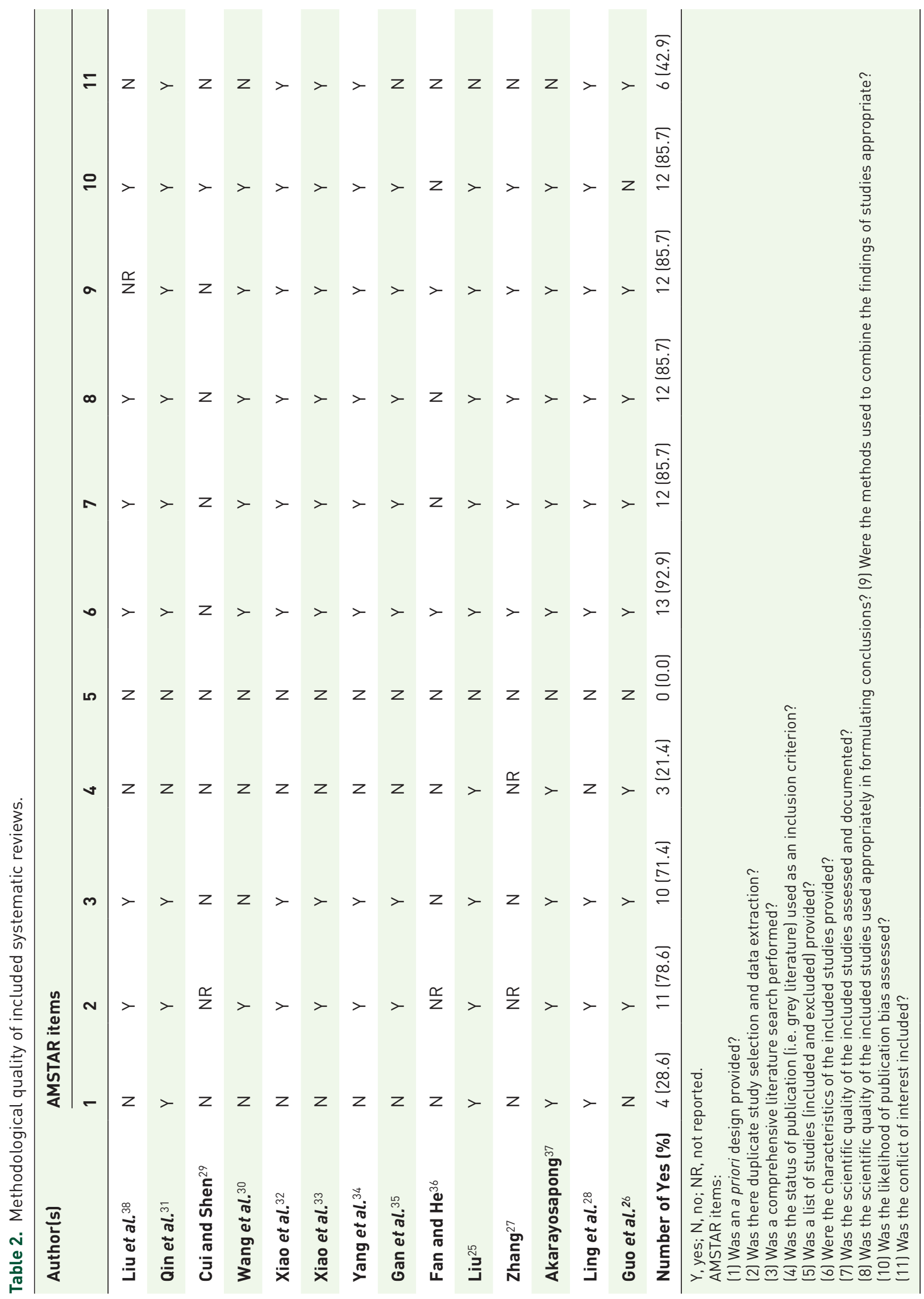


Table 3. Chinese herbal medicine for functional dyspepsia: systematic review of meta-analysis results.

\begin{tabular}{|c|c|c|c|c|}
\hline Author(s) & $\begin{array}{l}\text { Studies, } n \\
\text { (patients, } n \text { ) }\end{array}$ & Comparison* & Pooled results $(95 \% \mathrm{CI})$ & $\begin{array}{l}\text { Heterogeneity } \\
I^{2}(\%)\end{array}$ \\
\hline \multicolumn{5}{|c|}{ Alleviation of global dyspeptic symptoms } \\
\hline Liu et al. ${ }^{38^{* *}}$ & 4 (309) & $\mathrm{CHM}$ versus prokinetic agents & RR: $1.02(0.90,1.15)$ & $62 \$$ \\
\hline Qin et al. ${ }^{31}$ & $5(649)$ & $\begin{array}{l}\text { Modified Xiao Yao San versus } \\
\text { pharmacotherapy }\end{array}$ & OR: $3.53(2.32,5.36)$ & 0 \\
\hline Cui'29 & 23 (2013) & CHM versus domperidone & RR: $1.20(1.15,1.25)$ & 14 \\
\hline Cui and Shen 29 & 16 (1533) & CHM versus mosapride & RR: $1.14(1.06,1.24)$ & $62^{\$}$ \\
\hline Wang et al. ${ }^{30}$ & 12 (1069) & CHM versus pharmacotherapy & OR: $3.20(2.27,4.51)$ & 0 \\
\hline Wang et al. ${ }^{30}$ & $2(120)$ & $\mathrm{CHM}$ versus mosapride & OR: $3.98(1.50,10.54)$ & 0 \\
\hline Wang et al. ${ }^{30}$ & $8(663)$ & CHM versus domperidone & OR: $2.99(1.93,4.63)$ & 0 \\
\hline Xiao et al. ${ }^{32}$ & $3(350)$ & $\begin{array}{l}\text { Liu Jun Zi decoction versus prokinetic } \\
\text { agents }\end{array}$ & OR: $1.99(0.71,5.60)$ & $63^{\$}$ \\
\hline Xiao et al. ${ }^{33}$ & 7 (507) & $\begin{array}{l}\text { Xiang Sha Liu Jun Zi decoction versus } \\
\text { prokinetic agents }\end{array}$ & OR: $3.29(1.99,5.43)$ & 0 \\
\hline Yang et al. ${ }^{34}$ & 8 (728) & $\begin{array}{l}\text { Modified Chai Hu Shu Gan powder } \\
\text { versus pharmacotherapy }\end{array}$ & RR: $1.22(1.13,1.32)$ & 0 \\
\hline Gan et al. ${ }^{35}$ & 9 (872) & $\begin{array}{l}\text { Ban Xia Xie Xin decoction versus } \\
\text { prokinetic agents }\end{array}$ & OR: $2.58(1.72,3.85)$ & 0 \\
\hline Fan and $\mathrm{He}^{36 \ddagger}$ & 9 (907) & $\begin{array}{l}\text { Da Li Tong granule versus prokinetic } \\
\text { agents }\end{array}$ & OR: $1.17(0.88,1.55)$ & 0 \\
\hline $\operatorname{Liu}^{25}$ & 37 (3339) & $\mathrm{CHM}$ versus pharmacotherapy & RR: $1.15(1.10,1.20)$ & $52 \$$ \\
\hline Zhang 27 & 20 (1998) & CHM versus pharmacotherapy & OR: $3.73(2.85,4.88)$ & 0 \\
\hline Akarayosapong 37 & $9(786)$ & $\begin{array}{l}\text { Liu Wei An Xiao capsule versus } \\
\text { pharmacotherapy }\end{array}$ & RR: $1.29(1.12,1.49)$ & $76^{\$}$ \\
\hline Ling et al. ${ }^{28}$ & 27 (2793) & Si Ni San versus pharmacotherapy & OR: $3.81(3.05,4.77)$ & 0 \\
\hline Qin et al. ${ }^{31}$ & 6 (423) & $\begin{array}{l}\text { Modified Xiao Yao San }+ \\
\text { pharmacotherapy } \\
\text { versus pharmacotherapy }\end{array}$ & OR: $4.71(2.69,8.25)$ & 0 \\
\hline Xiao et al. ${ }^{33}$ & 9 (918) & $\begin{array}{l}\text { Shu Gan Jie Yu + mosapride versus } \\
\text { mosapride }\end{array}$ & OR: $3.57(2.53,5.05)$ & 0 \\
\hline Yang et al..$^{34}$ & $6(470)$ & $\begin{array}{l}\text { Modified Chai Hu Shu Gan powder } \\
+ \text { pharmacotherapy versus } \\
\text { pharmacotherapy }\end{array}$ & RR: 1.15 (1.07, 1.23) & 0 \\
\hline $\operatorname{Liu}^{25}$ & 8 (827) & $\begin{array}{l}\mathrm{CHM}+\text { pharmacotherapy versus } \\
\text { pharmacotherapy }\end{array}$ & RR: $1.23(1.14,1.34)$ & 28 \\
\hline Akarayosapong ${ }^{37}$ & $5(681)$ & $\begin{array}{l}\text { Liu Wei An Xiao capsule }+ \\
\text { pharmacotherapy versus } \\
\text { pharmacotherapy }\end{array}$ & RR: $1.22(1.14,1.32)$ & 0 \\
\hline
\end{tabular}


Table 3. (Continued)

\begin{tabular}{|c|c|c|c|c|}
\hline Author(s) & $\begin{array}{l}\text { Studies, } n \\
\text { (patients, } n \text { ) }\end{array}$ & Comparison* & Pooled results $(95 \% \mathrm{CI})$ & $\begin{array}{l}\text { Heterogeneity } \\
I^{2}(\%)\end{array}$ \\
\hline $\operatorname{Liu}^{25}$ & $4(459)$ & CHM versus placebo & RR: $1.47(1.29,1.68)$ & 0 \\
\hline Guo et al. ${ }^{26}$ & $7(1074)$ & $\mathrm{CHM}$ versus placebo & RR: $1.45(1.31,1.60)$ & 8 \\
\hline \multicolumn{5}{|c|}{ Alleviation of individual dyspeptic symptoms ${ }^{* *}$} \\
\hline \multicolumn{5}{|c|}{ (a) Alleviation of fullness sensation } \\
\hline Liu et al. ${ }^{38}$ & $1(55)$ & $\mathrm{CHM}$ versus domperidone & RR: $1.07(0.93,1.24)$ & NA \\
\hline \multicolumn{5}{|c|}{ (b) Gastric emptying \# } \\
\hline $\operatorname{Liu}^{25}$ & $1(16)$ & $\mathrm{CHM}$ versus pharmacotherapy & RR: $0.80(0.33,1.92)$ & NA \\
\hline Akarayosapong ${ }^{37}$ & $4(357)$ & $\begin{array}{l}\text { Liu Wei An Xiao capsule versus } \\
\text { prokinetic agents }\end{array}$ & MD: $2.16(0.26,4.17)$ & 4 \\
\hline \multicolumn{5}{|c|}{ (c) Alleviation of epigastric pain ${ }^{* *}$} \\
\hline Akarayosapong ${ }^{37}$ & $6(N R)$ & $\begin{array}{l}\text { Liu Wei An Xiao capsule versus } \\
\text { pharmacotherapy }\end{array}$ & RR: $1.18(1.06,1.31)$ & 28 \\
\hline \multicolumn{5}{|c|}{ (d) Alleviation of epigastric burning ${ }^{* *}$} \\
\hline Akarayosapong ${ }^{37}$ & $3(N R)$ & $\begin{array}{l}\text { Liu Wei An Xiao capsule versus } \\
\text { pharmacotherapy }\end{array}$ & RR: $0.98(0.76,1.26)$ & 0 \\
\hline \multicolumn{5}{|c|}{ (e) Alleviation of postprandial fullness ${ }^{* *}$} \\
\hline Akarayosapong ${ }^{37}$ & $3(N R)$ & $\begin{array}{l}\text { Liu Wei An Xiao capsule versus } \\
\text { pharmacotherapy }\end{array}$ & RR: $1.06(0.97,1.16)$ & 46 \\
\hline \multicolumn{5}{|c|}{ (f) Alleviation of early satiety ${ }^{* *}$} \\
\hline Akarayosapong ${ }^{37}$ & $5(N R)$ & $\begin{array}{l}\text { Liu Wei An Xiao capsule versus } \\
\text { pharmacotherapy }\end{array}$ & RR: $1.17(1.04,1.32)$ & 18 \\
\hline \multicolumn{5}{|l|}{ Quality of life } \\
\hline $\operatorname{Liu}^{25 I I}$ & $3(367)$ & CHM versus placebo & MD: $37.87(20.01,55.73)$ & 44 \\
\hline Guo et al. ${ }^{2611}$ & $6(698)$ & CHM versus placebo & SMD: $0.30(0.15,0.45)$ & 0 \\
\hline $\operatorname{Liu}^{25 I I}$ & 5 (387) & $\mathrm{CHM}$ versus pharmacotherapy & MD: $4.82(2.13,7.50)$ & $98^{\$}$ \\
\hline \multicolumn{5}{|c|}{ Alleviation of Chinese medicine syndrome } \\
\hline Guo et al. ${ }^{26}$ & $5(741)$ & CHM versus placebo & RR: $1.36(1.23,1.50)$ & 31 \\
\hline \multicolumn{5}{|c|}{ Plasma acylated ghrelin level } \\
\hline Xiao et al. ${ }^{32}$ & $1(27)$ & Liu Jun Zi Tang versus domperidone & MD: $9.00(-0.27,18.27)$ & NA \\
\hline \multicolumn{5}{|c|}{ 'Obviously effective' rate $\ddagger$} \\
\hline Fan and $\mathrm{He}^{36}$ & 9 (907) & $\begin{array}{l}\text { Da Li Tong granule versus prokinetic } \\
\text { agents }\end{array}$ & OR: $1.38(0.90,2.11)$ & 0 \\
\hline Safetyף & & & & \\
\hline
\end{tabular}


Table 3. (Continued)

\begin{tabular}{|c|c|c|c|c|}
\hline Author(s) & $\begin{array}{l}\text { Studies, } n \\
\text { (patients, } n \text { ) }\end{array}$ & Comparison* & Pooled results $(95 \% \mathrm{CI})$ & $\begin{array}{l}\text { Heterogeneity } \\
\rho^{2}(\%)\end{array}$ \\
\hline Liu $^{25}$ & 9 (761) & $\mathrm{CHM}$ versus pharmacotherapy & OR: $0.98(0.45,2.12)$ & 0 \\
\hline Zhang 27 & 4 (388) & $\mathrm{CHM}$ versus pharmacotherapy & OR: $0.14(0.03,0.63)$ & 0 \\
\hline Akarayosapong ${ }^{37}$ & $6(425)$ & $\begin{array}{l}\text { Liu Wei An Xiao capsule versus } \\
\text { pharmacotherapy }\end{array}$ & OR: $1.11(0.38,3.23)$ & 0 \\
\hline $\operatorname{Liu}^{25}$ & 2 (222) & $\begin{array}{l}\mathrm{CHM}+\text { pharmacotherapy versus } \\
\text { pharmacotherapy }\end{array}$ & OR: $1.22(0.30,4.89)$ & 0 \\
\hline Guo et al. ${ }^{26}$ & $4(771)$ & CHM versus placebo & RR: $1.06(0.66,1.70)$ & 0 \\
\hline \multicolumn{5}{|c|}{$\begin{array}{l}\text { *CHM denotes the inclusion of multiple types of Chinese herbal medicine. The generic term 'prokinetic agents' is used to denote that multiple types } \\
\text { of prokinetic agents were included in the comparison. The term 'pharmacotherapy' was used when both proton pump inhibitor and prokinetic } \\
\text { agents were included in the comparison. If the authors evaluated multiple CHM formulations and provided detailed information, we reported the } \\
\text { compositions of herbal formulations in Table } \mathrm{A} 3 \text { in the appendix. }\end{array}$} \\
\hline \multicolumn{5}{|c|}{$\begin{array}{l}\text { **Alleviation of global or individual dyspeptic symptoms is measured by Gastrointestinal Symptom Rating Scale (GSRS) or other standardized } \\
\text { scales. GSRS is a score in which patients report dyspeptic symptom severity on a four-point Likert scale (symptom free, marked improvement, } \\
\text { slight improvement, and no improvement). }\end{array}$} \\
\hline \multicolumn{5}{|c|}{$\$ p<0.10$ for the heterogeneity test. } \\
\hline \multicolumn{5}{|c|}{$\begin{array}{l}\text { ¥'Obviously effective' rate denotes a numerical result } \geqslant 60 \% \text { in the following calculation: [(Severity score of symptoms before treatment }- \text { Severity } \\
\text { score of symptoms after treatment] / Severity score of symptoms before treatment] } \times 100 \% \text {, with symptoms measured by the GSRS and other } \\
\text { standardized scales. }\end{array}$} \\
\hline \multicolumn{5}{|c|}{$\S$ Quality of life is measured with the 36-Item Short Form Survey (SF-36). } \\
\hline \multicolumn{5}{|c|}{$\begin{array}{l}\text { "Quality of life is measured with the 36-Item Short Form Survey (SF-36), Quality of Life Questionnaire for Functional Digestive Disorders, } \\
\text { Functional Dyspepsia-Related Quality of Life or other validated scales. }\end{array}$} \\
\hline \multicolumn{5}{|c|}{$\begin{array}{l}\text { "Safety denotes the occurrence of any side effects or adversities due to the use of interventions or placebo. This definition applies to all results } \\
\text { under the 'Safety' section in Table } 3 \text {. }\end{array}$} \\
\hline \multicolumn{5}{|c|}{$\begin{array}{l}\text { \#Gastric emptying rate is defined as the GSRS scores in emptying the stomach }{ }^{25} \text { and as the percentage of substances remaining in stomach } 4-5 \mathrm{~h} \\
\text { after a meal. }{ }^{37}\end{array}$} \\
\hline \multicolumn{5}{|c|}{$\begin{array}{l}\ddagger \ddagger \text { Alleviation of Chinese medicine syndrome measures symptom improvement according to traditional Chinese medical theories and standards. } \\
\text { CHM, Chinese herbal medicine; Cl, confidence interval; RR, risk ratio; OR, odds ratio; MD, mean difference; SMD, standard mean difference; NA, } \\
\text { not applicable. }\end{array}$} \\
\hline
\end{tabular}

an add-on to pharmacotherapy (1 comparison). Table 4 presents a summary of the main findings in this SR.

For the control arm, eight comparisons summarized evidence from specific prokinetic agents, namely mosapride (five comparisons) and domperidone (two comparisons). The remaining one comparison combined the outcome using mosapride or domperidone against $\mathrm{CHM}$ to generate a pooled result. On the other hand, 14 comparisons summarized evidence from multiple types of pharmacotherapy, either comparing CHM + pharmacotherapy with pharmacotherapy (4 comparisons) or comparing CHM with pharmacotherapy (10 comparisons). There are also two comparisons between multiple types of CHM and placebo of CHM on global symptom improvement. Details on the results are listed in Table 3.
Chinese herbal medicine versus pharmacotherapy. In total, there are 17 pooled results on this comparison, either including multiple types of CHM formulae (8 comparisons) or focusing on a single CHM (9 comparisons). Results showed that CHM is generally superior to pharmacotherapy in improving FD global symptoms, as 13 out of 17 comparisons revealed that CHM had higher treatment effectiveness than pharmacotherapy on FD. Among the eight pooled results that included multiple types of CHM formulae, the effect size ranged from a pooled OR of 3.98 (95\% CI $=1.50-10.54, I^{2}=0 \%$, two RCTs) to a pooled RR of $1.02\left(95 \% \mathrm{CI}=0.90-1.15, I^{2}=\right.$ $62 \%$, four RCTs).

There were three specific CHM formulae which appeared to show larger effect sizes. They are $\mathrm{Si}$ Ni San (pooled OR $=3.81,95 \% \mathrm{CI}=3.05-4.77$, $I^{2}=0 \%, 27$ RCTs), modified Xiao Yao San 
Table 4. Chinese herbal medicine for functional dyspepsia: summary of findings for the main comparison.

\begin{tabular}{|c|c|c|c|c|}
\hline \multicolumn{5}{|c|}{ Chinese herbal medicine compared with pharmacotherapy for functional dyspepsia } \\
\hline \multicolumn{5}{|c|}{$\begin{array}{l}\text { Population: Patients with functional dyspepsia } \\
\text { Intervention: Chinese herbal medicine } \\
\text { Comparison: Pharmacotherapy } \\
\text { Settings: Any }\end{array}$} \\
\hline \multirow[t]{2}{*}{ Outcomes } & \multicolumn{2}{|l|}{$\begin{array}{l}\text { Illustrative } \\
\text { comparative risks } \\
(95 \% \mathrm{CI})\end{array}$} & \multirow[t]{2}{*}{$\begin{array}{l}\text { Relative effect } \\
(95 \% \text { CI) }\end{array}$} & \multirow[t]{2}{*}{$\begin{array}{l}\text { Participants, } n \\
\text { (studies, } n \text { ) }\end{array}$} \\
\hline & Assumed risk & $\begin{array}{l}\text { Corresponding } \\
\text { risk }\end{array}$ & & \\
\hline \multirow{2}{*}{$\begin{array}{l}\text { Alleviation of } \\
\text { global dyspeptic } \\
\text { symptoms }\end{array}$} & Pharmacotherapy & $\begin{array}{l}\text { Modified Xiao Yao } \\
\text { San }\end{array}$ & $\begin{array}{l}\text { OR: } 3.53 \text { (2.32, } \\
5.36)\end{array}$ & $649(5)$ \\
\hline & 694 per 1000 & 890 per 1000 & & \\
\hline \multirow{2}{*}{$\begin{array}{l}\text { Alleviation of } \\
\text { global dyspeptic } \\
\text { symptoms }\end{array}$} & Prokinetic agents & $\begin{array}{l}\text { Xiang Sha Liu Jun } \\
\text { Zi decoction }\end{array}$ & $\begin{array}{l}\text { OR: } 3.29 \text { (1.99, } \\
5.43)\end{array}$ & $507(7)$ \\
\hline & 740 per 1000 & 934 per 1000 & & \\
\hline \multicolumn{5}{|c|}{ Chinese herbal medicine + pharmacotherapy compared with pharmacotherapy for functional dyspepsia } \\
\hline \multicolumn{5}{|c|}{$\begin{array}{l}\text { Population: Patients with functional dyspepsia } \\
\text { Intervention: Chinese herbal medicine }+ \text { pharmacotherapy } \\
\text { Comparison: Pharmacotherapy } \\
\text { Settings: Any }\end{array}$} \\
\hline \multirow[t]{3}{*}{ Outcomes } & \multicolumn{2}{|l|}{$\begin{array}{l}\text { Illustrative } \\
\text { comparative risks } \\
(95 \% \mathrm{CI})\end{array}$} & \multirow[t]{2}{*}{$\begin{array}{l}\text { Relative effect } \\
(95 \% \text { CI) }\end{array}$} & \multirow[t]{2}{*}{$\begin{array}{l}\text { Participants, } n \\
\text { (studies, } n \text { ) }\end{array}$} \\
\hline & Assumed risk & $\begin{array}{l}\text { Corresponding } \\
\text { risk }\end{array}$ & & \\
\hline & Pharmacotherapy & $\begin{array}{l}\mathrm{CHM}+ \\
\text { pharmacotherapy }\end{array}$ & & \\
\hline \multirow[t]{2}{*}{$\begin{array}{l}\text { Alleviation of } \\
\text { global dyspeptic } \\
\text { symptoms }\end{array}$} & Pharmacotherapy & $\begin{array}{l}\text { Modified Xiao } \\
\text { Yao San }+ \\
\text { pharmacotherapy }\end{array}$ & $\begin{array}{l}\text { OR: } 4.71 \text { (2.69, } \\
8.25)\end{array}$ & $423(6)$ \\
\hline & 705 per 1000 & 917 per 1000 & & \\
\hline \multirow[t]{2}{*}{$\begin{array}{l}\text { Alleviation of } \\
\text { global dyspeptic } \\
\text { symptoms }\end{array}$} & Mosapride & $\begin{array}{l}\text { Shu Gan Jie } \\
\text { Yu capsule }+ \\
\text { mosapride }\end{array}$ & $\begin{array}{l}\text { OR: } 3.57 \text { (2.53, } \\
5.05)\end{array}$ & 918 (9) \\
\hline & 678 per 1000 & 880 per 1000 & & \\
\hline \multicolumn{5}{|c|}{$\begin{array}{l}\text { Alleviation of global or individual dyspeptic symptoms is measured by the Gastrointestinal Symptom Rating Scale (GSRS } \\
\text { or other standardized scales. GSRS measures patient reported dyspeptic symptom severity on a four-point Likert scal } \\
\text { (symptom free, marked improvement, slight improvement, and no improvement). } \\
\text { CHM, Chinese herbal medicine; OR, odds ratio; Cl, confidence interval. }\end{array}$} \\
\hline
\end{tabular}


(pooled OR $=3.53,95 \% \mathrm{CI}=2.32-5.36, I^{2}=$ $0 \%, 5$ RCTs) and Xiang Sha Liu Jun Zi decoction (pooled OR $=3.29,95 \% \mathrm{CI}=1.99-5.43, I^{2}$ $=0 \%, 7$ RCTs). Heterogeneity was low across all three meta-analyses.

Chinese herbal medicine plus pharmacotherapy versus pharmacotherapy. A total of five SRs reported pooled results of this comparison and four of them restricted the type of CHM formula used. With the combined use of CHM and pharmacotherapy, the add-on effectiveness was obvious, as all of the summarized evidence favored the combined treatment group. Modified Xiao Yao San has a relatively better performance, with pooled $\mathrm{OR}=$ 4.71 (95\% CI $=2.69-8.25, I^{2}=0 \%$, six RCTs).

Chinese herbal medicine versus placebo. There were two pooled results summarized from 11 RCTs comparing CHM with placebo on the effectiveness on FD. Both showed that CHM was more effective than placebo, with pooled RR being 1.45 (95\% CI $=1.31-1.60, I^{2}=8 \%$, seven RCTs) and 1.47 (95\% CI $=1.29-1.68, I^{2}=0 \%$, four RCTs), respectively. Both of them did not restrict the types of CHM formula in the analyses.

\section{Alleviation of individual dyspeptic symptoms}

Alleviation of fullness sensation. There is one RCT comparing CHM and domperidone on this outcome, showing that CHM performed slightly better in reducing fullness sensation, but the difference did not reach significance (RR: $1.07,95 \%$ $\mathrm{CI}=0.93-1.24,55$ patients).

Gastric emptying. There were two meta-analyses on this outcome. One demonstrated that Liu Wei An Xiao capsule had a better gastric emptying effect than prokinetic agents (pooled $\mathrm{MD}=2.16,95 \% \mathrm{CI}$ $=0.26-4.17, I^{2}=4 \%$, four RCTs). The other one comparing $\mathrm{CHM}$ and pharmacotherapy found no significant difference between the two interventions.

Alleviation of epigastric pain. One meta-analysis based on six RCTs revealed that Liu Wei An Xiao capsule was more favorable than pharmacotherapy in reducing epigastric pain, with a pooled $\mathrm{RR}=1.18\left(95 \% \mathrm{CI}=1.06-1.31, I^{2}=28 \%\right)$.

Alleviation of epigastric burning. One metaanalysis based on three RCTs showed that there was no significant difference between Liu Wei An Xiao capsule and prokinetic agents, with a pooled $\mathrm{RR}=0.98\left(95 \% \mathrm{CI}=0.76-1.26, I^{2}=0 \%\right)$.
Alleviation of postprandial fullness. One metaanalysis summarized from three RCTs revealed that there was no significant difference between Liu Wei An Xiao capsule and pharmacotherapy in alleviation of postprandial fullness, with a pooled $\mathrm{RR}=1.06\left(95 \% \mathrm{CI}=0.97-1.16, I^{2}=46 \%\right)$.

Alleviation of early satiety. One meta-analysis from five RCTs demonstrated that Liu Wei An Xiao capsule could relieve early satiety better than prokinetic agents $($ pooled $\mathrm{RR}=1.17,95 \% \mathrm{CI}=$ $\left.1.04-1.32, I^{2}=18 \%\right)$.

\section{Secondary outcomes}

Quality of life. Three pooled results were reported from two SRs, which did not restrict the use of particular CHM formula. They all showed that CHM improved the quality-of-life scoring of patients to a greater extent as compared with pharmacotherapy or placebo. The most significant pooled $\mathrm{MD}$ is 37.87 ( $95 \% \mathrm{CI}=20.01-$ 55.73, $I^{2}=44 \%$, three RCTs), but moderate heterogeneity is present in this meta-analysis.

Alleviating Chinese medicine syndrome severity. One meta-analysis derived from five RCTs demonstrated that CHM was more effective in reducing severity of Chinese medicine syndrome than placebo, with pooled $\mathrm{RR}=1.36(95 \% \mathrm{CI}=$ $\left.1.23-1.50, I^{2}=31 \%\right)$.

Safety. Five SRs reported pooled results for safety, with three comparing CHM with pharmacotherapy, one comparing CHM plus pharmacotherapy with pharmacotherapy alone, and the remaining one comparing CHM with placebo. Four pooled results showed that there were no significant differences between the treatment and control groups in causing adverse events. The remaining one reported that $\mathrm{CHM}$ was safer than pharmacotherapy, with pooled OR of $0.14(95 \%$ CI $=0.03-0.63, I^{2}=0 \%$, four RCTs) (Table 3). Adverse events that could be attributed to CHM treatment include epigastric discomfort, increased passing of flatus, abdominal fullness and more frequent egestion. Details on reported adverse events are shown in Table A3 in the appendix.

\section{Discussion}

Evidence from included SRs showed that CHM outperformed pharmacotherapy at improving global and individual dyspeptic symptoms. Three 
specific CHM formulae which appeared to show superior results in the alleviation of global dyspeptic symptoms are Si Ni San, modified Xiao Yao San and Xiang Sha Liu Jun Zi decoction. Taking into consideration the safety of using CHM, available evidence suggested that CHM might be safer than pharmacotherapy. Moreover, even if negative effects occurred, they were mild and tolerated by the patients. Therefore, CHM could be an alternative, or used as an add-on treatment, to pharmacotherapy. Yet, future trials should address methodological shortcomings of existing trials. Many trials contributing to the SR result had unclear risk of bias in terms of sequence generation, allocation concealment and blinding. To improve the reporting quality, future randomized controlled trials should also comply with the CONSORT reporting guideline. ${ }^{50}$

Among the total of 14 SRs, we identified 8 SRs that used specific CHM formulae as intervention, while the remaining six did not restrict their CHM formulae. When there is no restriction on the $\mathrm{CHM}$ formulae, the results could only indicate the general effectiveness of CHM. The applicability of such results is limited, as the generalized results from different formulae did not indicate their comparative effectiveness. Therefore, it is difficult to conclude which formula has the highest probability in improving different FD symptoms. Future network meta-analysis and follow-up trials on the most promising CHM formula should be conducted. ${ }^{51}$

In routine practice, the prescription of Chinese herbal medicine is often tailored in accordance to the patient's Chinese medicine syndrome type, which is diagnosed via the 'four diagnostic procedure' in Chinese medicine: inspection, auscultation and olfaction, inquiry and palpation. However, only five $e^{30,34,36-38}$ of the 14 SRs considered Chinese medicine syndrome type as a trial eligibility criterion. In order words, more than $60 \%$ of the results reported in this SR only considered the use of CHM for managing FD, without additional consideration of aligning Chinese medicine syndrome type and choice of CHM. This provides a potential explanation on variations in effect sizes reported in different metaanalyses and trials. In improved model validity of future trials, investigators should incorporate Chinese medicine diagnosis into the inclusion criteria. Nevertheless, standardizing Chinese medicine diagnosis for FD has proved difficult, ${ }^{52}$ but such obstacles could be resolved by group consensus technique or emerging machine learning methods. ${ }^{53,54}$

During the process of literature selection, we observed a noteworthy problem among the SRs, which is a lack of detailed description of the outcome measurement method. We have therefore excluded 16 SRs from our SR of SRs for this reason. Without a validated outcome measurement approach, the data derived would become hard to interpret. This common flaw should be rectified in future research by providing information on the validity, reliability and responsiveness of the outcome measurement scale or approach. With regards to outcome selection, both objectives, as well as validated patient-centered outcomes should be reported in future trials, including: (a) individual symptom assessment; (b) disease-specific quality of life questionnaire; (c) nutrient drink test; and (d) gastric emptying test. ${ }^{55}$ Also, follow-up duration of our included RCTs was only 2-8 weeks. Longer-term benefits of CHM should be evaluated by following the recommended follow-up duration of at least 12 weeks. ${ }^{55}$

Also, the external validity of our results is limited by the use of heterogeneous diagnostic criteria for inclusion in different trials. Rome III has been adopted in more than half of the included RCTs, and its application is limited by the fact that it may lead to exclusion of a substantial number of patients with endoscopically verified FD. ${ }^{56}$ The application of such a strict inclusion criteria implies that trial patients are likely to differ from the average patient seen in clinical practice. ${ }^{56}$ In the newly released Rome IV criteria, only minor modifications were made with regards to symptom description ${ }^{57}$ and this problem could persist if future trials continue to adopt Rome IV criteria. In future, more flexible diagnostic criteria might be used in recruiting patients in FD clinical trials. ${ }^{58}$ Additionally, since the evidence summarized was obtained from reports published in China, we should take note of the potential publication bias. ${ }^{59,60}$ All participants from our included trials were Chinese, the applicability of our results to other ethnicities is therefore limited.

Among the included SRs, CHM has demonstrated a good safety profile, with adverse drug reactions (ADRs) induced by herbal medicine being generally mild. However, existing evidence has suggested that certain CHM may induce 
more series adverse events, ${ }^{61-64}$ which highlights the importance of supervision when CHM is prescribed for the treatment of FD.

Finally, it should be highlighted that the methodological quality of SRs regarding CHM is mediocre. Future SR teams should be providing lists of included and excluded studies, and including grey literature in their synthesis. Also, reporting quality of the SRs is less than satisfactory. Only 2 out of the 14 SRs reported the duration of FD diagnosis, which is an importing piece of information for guiding the choice of appropriate treatment for patients with different duration of diagnosis. Six of them did not report details of treatment regimens, including the frequency and dosage. This hinders the translation of research findings into daily practice. It is also noteworthy that some meta-analysis pooled trial data used OR instead of RR. This is not a preferred practice, as RR should be use for quantifying RCT results. The use of OR would lead to exaggeration of effect sizes and readers should be cautious when interpreting these results. ${ }^{65}$

In conclusion, current clinical evidence has revealed that CHM can be considered an option for FD treatment. Future trials can focus on the improvement of individual dyspeptic symptoms, including PDS and EPS, together with global symptom improvement, in order to provide a more comprehensive and detailed analysis. In addition, the methodological and reporting qualities of future SRs and clinical trials have to be improved urgently, with adherence to international standards.

\section{Funding}

This research received no specific grant from any funding agency in the public, commercial, or notfor-profit sectors.

\section{Conflict of interest statement}

The authors declare that there is no conflict of interest.

\section{References}

1. Kumar A, Pate J and Sawant P. Epidemiology of functional dyspepsia. $\mathcal{F}$ Assoc Physicians India 2012; 60(Suppl.): 9-12.

2. El-Serag HB and Talley NJ. Systemic review: the prevalence and clinical course of functional dyspepsia. Aliment Pharmacol Ther 2004; 19: 643-654.

3. Ghoshal U, Singh R, Chang FY, et al. Epidemiology of uninvestigated and functional dyspepsia in Asia: facts and fiction. $\mathcal{F}$ Neurogastroenterol Motil 2011; 17: 235-244.

4. Kumar D, Bhat ZA, Singh P, et al. Ailanthus excelsa Roxb is really a plant of heaven. Int $\mathcal{F}$ Pharmacol 2010; 6: 535-550.

5. Piessevaux H, De Winter B, Louis E, et al. Dyspeptic symptoms in the general population: a factor and cluster analysis of symptom groupings. Neurogastroenterol Motil 2009; 21: 378-388.

6. Welen K, Faresjo A and Faresjo T. Functional dyspepsia affects women more than men in daily life: a case-control study in primary care. Gened Med 2008; 5: 62-73.

7. Suzuki H. The application of the Rome IV criteria to functional esophagogastroduodenal disorders in Asia. $\mathcal{F}$ Neurogastroenterol Motil 2017; 23: 325-333.

8. Tack J, Talley NJ, Camilleri M, et al. Functional gastroduodenal disorders. Gastroenterology 130: 1466-1479.

9. Camilleri M and Stanghellini V. Current management strategies and emerging treatments for functional dyspepsia. Nat Rev Gastroenterol Hepatol 2013; 10: 187-194.

10. Sundberg R, Palmqvist M, Tunsäter A, et al. Health-related quality of life in young adults with asthma. Respir Med 2009; 103: 1580-1585.

11. Lix L, Graff LA, Walker JR, et al. Longitudinal study of quality of life and psychological functioning for active, fluctuating, and inactive disease patterns in inflammatory bowel disease. Inflamm Bowel Dis 2008; 14: 1575-1584.

12. Lacy B, Weiser KT, Kennedy AT, et al. Functional dyspepsia: the economic impact to patients. Aliment Pharmacol Ther 2013; 38: 170-177.

13. Malfertheiner P, Megraud F, O'Morain CA, et al. Management of Helicobacter pylori infection-the Maastricht IV/ Florence consensus report. Gut 2012; 61: 646-664.

14. Moayyedi P, Soo S, Deeks J, et al. Pharmacological interventions for non-ulcer dyspepsia. Cochrane Database Syst Rev 2006; (2): CD001960.

15. Xiao YL, Peng S, Tao J, et al. Prevalence and symptom pattern of pathologic esophageal acid reflux in patients with functional dyspepsia based 
on the Rome III criteria. Am $\mathcal{F}$ Gastroenterol 2010; 105: 2626-2631.

16. Rosch W, Liebregts T, Gundermann KJ, et al. Phytotherapy for functional dyspepsia: a review of the clinical evidence for the herbal preparation STW 5. Phytomedicine. 2006; 13(Suppl. 5): 114-121.

17. Hojo M, Nagahara A, Asaoka D, et al. Emerging pharmacological therapy for functional dyspepsia. Clin F Gastroenterol 2013; 6: 352-356.

18. Suzuki H, Okada S and Hibi T. Proton-pump inhibitors for the treatment of functional dyspepsia. Therap Adv Gastroenterol 2011; 4: 219-226.

19. Quigley EMM. Prokinetics in the Management of Functional Gastrointestinal Disorders. J Neurogastroenterol Motil 2015; 21: 330-336.

20. Leelakanok N, Holcombe A and Schweizer ML. Domperidone and risk of ventricular arrhythmia and cardiac death: a systematic review and meta-analysis. Clin Drug Investig 2016; 36: 97-107.

21. Shin HW and Chung SJ. Drug-induced parkinsonism. f Clin Neurol 2012; 8: 15-21.

22. Bosco D, Plastino M, Marcello MG, et al. Acute hemifacial dystonia possibly induced by clebopride. Clin Neuropharmacol 2009; 32: 107-108.

23. Spleen and Stomach Diseases Committee CAoCM. Consensus of diagnosis and treatment of Chinese medicine on dyspepsia. Chin F Integ Med 2009; 30: 533-537.

24. Zheng XY. Guiding principle of clinical research on new drugs of traditional Chinese Medicine. Beijing: China Medic-Pharmaceutical Sciences and Technology Publishing House, 2002, p.143.

25. Liu PF. Systematic review of Chinese medicine in treating functional dyspepsia. China: Guangxi Medical University, 2015.

26. Guo Y, Zhu JJ, Su XL, et al. Efficacy of Chinese herbal medicine in functional dyspepsia: a metaanalysis of randomized, double-blind, placebocontrolled trials. $\mathcal{F}$ Tradit Chin Med Sci 2016; 3: 147-156.

27. Zhang SZ. Systematic review on acrid opening and bitter downbearing method in treating functional dyspepsia. China: Shaanxi University of Chinese Medicine, 2015.

28. Ling W, Li Y, Jiang W, et al. Common mechanism of pathogenesis in gastrointestinal diseases implied by consistent efficacy of single
Chinese medicine formula. Medicine (United States) 2015; 94: e1111.

29. Cui YP and Shen H. Meta-analysis of Chinese Medicine in Treating Functional Dyspepsia. World federation of Chinese medicine societies digestive disease professional committee $3 \mathrm{rd}$ international academic conference cum Guangxi society of integrated traditional Chinese and western medicine digestive disease annual conference proceedings. Nanning, China, 2012, p.1-8.

30. Wang C, Zhu M, Xia W, et al. Meta-analysis of traditional Chinese medicine in treating functional dyspepsia of liver-stomach disharmony syndrome. F Tradit Chin Med 2012; 32: 515-522.

31. Qin F, Huang X and Ren P. Chinese herbal medicine modified Xiao Yao San for functional dyspepsia: meta-analysis of randomized controlled trials. $\mathcal{F}$ Gastroenterol Hepatol (Australia). 2009; 24: 1320-1325.

32. Xiao Y, Liu YY, Yu KQ, et al. Chinese herbal medicine Liu Jun Zi Tang and Xiang Sha Liu Jun $\mathrm{Zi}$ Tang for functional dyspepsia: meta-analysis of randomized controlled trials. Evid Based Complement Alternat Med. Epub ahead of print 12 December 2012. DOI: 10.1155/2012/936459.

33. Xiao ZJ, Guo JW and Xu F. Efficacy of Chinese medicine Shu Gan Jie Yu capsule for functional dyspepsia. Acta Pharmacologica Sinica 2013; 34: 23.

34. Yang N, Jiang X, Qiu X, et al. Modified Chai Hu Shu Gan powder for functional dyspepsia: metaanalysis for randomized controlled trial. Evid Based Complement Alternat Med 2013; 2013: no pagination.

35. Gan Y, Liu H, Yang L, et al. Effect of Ban Xia Xie Xin Tang on treatment of functional dyspepsia: a meta-analysis of randomized controlled trials. $\mathcal{F}$ Tradit Chin Med 2014; 34: 140-144.

36. Fan CS and He MM. Cost-effectiveness analysis and evaluation of $\mathrm{Da} \mathrm{Li}$ Tong granule in treating functional dyspepsia. Eval Med 2015; 6: 24-34.

37. Akarayosapong P. Systematic review on the effectiveness and safety of Liu Wei An Xiao Capsule in treating functional dyspepsia. China: Guangxi Medical University, 2015.

38. Liu JL, Zhang YJ, Song YX, et al. Systematic review on randomized control trials of Chinese and Western medicine in treating functional dyspepsia. Chin F Evid Based Med 2009; 9: 1315-1322. 
39. Wysowski D, Corken A, Gallo-Torres H, et al. Postmarketing reports of QT prolongation and ventricular arrhythmia in association with cisapride and Food and Drug Administration regulatory actions. Am F Gastroenterol 2001; 96: 1698-1703.

40. Ren L, Chen WX, Qian LJ, et al. Addition of prokinetics to PPI therapy in gastroesophageal reflux disease: a meta-analysis. World $\mathcal{F}$ Gastroenterol 2014; 20: 2412-2419.

41. Eom CS, Jeon CY, Lim JW, et al. Use of acidsuppressive drugs and risk of pneumonia: a systematic review and meta-analysis. $C M A \mathcal{F}$ 2011; 183: 310-319.

42. Lam J, Schneider JL, Zhao W, et al. Proton pump inhibitor and histamine 2 receptor antagonist use and vitamin B12 deficiency. $\mathcal{F A M A} 2013 ; 310$ : 2435-2442.

43. Hanlon J, Landerman LR, Artz MB, et al. Histamine 2 receptor antagonist use and decline in cognitive function among community dwelling elderly. Pharmacoepidemiol Drug Saf 2004; 13: 781-787.

44. Haynes R, McKibbon KA, Wilczynski NL, et al. Optimal search strategies for retrieving scientifically strong studies of treatment from Medline: analytical survey. BMF 2005; 330: 1179.

45. Wong SSL, Wilczynski NL and Haynes RB. Developing optimal search strategies for detecting clinically sound treatment studies in EMBASE. $\mathcal{F}$ Med Libr Assoc 2006; 94: 41-47.

46. Shea BJ, Hamel C, Wells GA, et al. AMSTAR is a reliable and valid measurement tool to assess the methodological quality of systematic reviews. f Clin Epidemiol 2009; 62: 1013-1020.

47. Pieper D, Buechter RB, Li L, et al. Systematic review found AMSTAR, but not R(evised)AMSTAR, to have good measurement properties. f Clin Epidemiol 2015; 68: 574-583.

48. Higgins JP and Green S. Cochrane handbook for systematic reviews of interventions. Hoboken, New Jersey: John Wiley \& Sons, 2011.

49. Higgins J, Thompson SG, Deeks JJ, et al. Measuring inconsistency in meta-analyses. BMF 2003; 327: 557-560.

50. Schulz K, Altman DG, Moher D, et al. CONSORT 2010 statement: updated guidelines for reporting parallel group randomised trials. BMF 2010; 340: c332.

51. Mills EJ, Thorlund $\mathrm{K}$ and Ioannidis JP. Demystifying trial networks and network metaanalysis. BMF 2013; 346: f2914.
52. Shuldiner SR, Chung VC, Wu X, et al. Methodological challenges in mapping chinese medicine syndrome with conventional diagnosis: implications for multi-centre trials in integrative medicine. Eur f Integr Med 2015; 7: 358-364.

53. Chung VC, Ho RS, Wu X, et al. Incorporating traditional Chinese medicine syndrome differentiation in randomized trials: methodological issues. Eur F Integr Med 2016; 8: 898-904.

54. Zhang NL, Fu C, Liu TF, et al. A data-driven method for syndrome type identification and classification in traditional Chinese medicine. $\mathcal{F}$ Integr Med 2017; 15: 110-123.

55. Lacy BE, Talley NJ and Camilleri M. Functional dyspepsia: time to change clinical trial design[quest]. Am F Gastroenterol 2010; 105: 2525-2529.

56. Van Kerkhoven L, Laheij RJ, Meineche-Schmidt $\mathrm{V}$, et al. Functional dyspepsia: not all roads seem to lead to Rome. $\mathcal{F}$ Clin Gastroenterol 2009; 43: 118-122.

57. Sood R and Ford AC. Diagnosis: Rome IV criteria for FGIDs - an improvement or more of the same? Nat Rev Gastroenterol Hepatol 2016; 13: 501-502.

58. Scott RS, Chung VCH, Wu XY, et al. Methodological challenges in mapping chinese medicine syndrome with conventional diagnosis: implications for multi-centre trials in integrative medicine. Eur F Integr Med 2015; 7: 358-364.

59. Tang JL, Zhan SY and Ernst E. Review of randomised controlled trials of traditional Chinese medicine. BMF 1999; 319: 160-161.

60. Vickers A, Goyal N, Harland R, et al. Do certain countries produce only positive results? A systematic review of controlled trials. Control Clin Trials 1998; 19: 159-166.

61. Zeng Z-P and Jiang J-G. Analysis of the adverse reactions induced by natural product-derived drugs. Br F Pharmacol 2010; 159: 1374-1391.

62. Meyer MM, Chen T-P and Bennett WM. Chinese herb nephropathy. Proc (Bayl Univ Med Cent). 2000; 13: 334-337.

63. Douros A, Bronder E, Andersohn F, et al. Herbinduced liver injury in the Berlin Case-Control Surveillance Study. Int F Mol Sci 2016; 17: 114.

64. Cho J-H, Oh D-S, Hong S-H, et al. A nationwide study of the incidence rate of herb-induced liver injury in Korea. Arch Toxicol 2017; 91: 4009-4015.

65. Viera AJ. Odds ratios and risk ratios: what's the difference and why does it matter? South Med $\mathcal{F}$ 2008; 101: 730-734. 


\section{Appendices}

Table A1. Search strategies and results for systematic reviews on Chinese herbal medicine for functional dyspepsia.

(1) Cochrane Database of Systematic Reviews from inception to 23 January 2017.

\begin{tabular}{|c|c|c|}
\hline 1 & dyspepsia*.mp. & 227 \\
\hline 2 & functional dyspepsia*.mp. & 15 \\
\hline 3 & non ulcer dyspepsia*.mp. & 11 \\
\hline 4 & 1 or 2 or 3 & 227 \\
\hline 5 & Chinese herb*.mp. & 209 \\
\hline 6 & herb*.mp. & 709 \\
\hline 7 & Traditional Chinese medic*.mp. & 243 \\
\hline 8 & phytother*.mp. & 137 \\
\hline 9 & (chinese adj5 (traditional or medic*).mp. & 500 \\
\hline 10 & (plant or plants).mp. & 407 \\
\hline 11 & (traditional adj5 medic*).mp. & 380 \\
\hline 12 & Chinese medic*.mp. & 374 \\
\hline 13 & oriental medic*.mp. & 30 \\
\hline 14 & herbaceous agent.mp. & 13 \\
\hline 15 & medicinal plant*.mp. & 34 \\
\hline 16 & 5 or 6 or 7 or 8 or 9 or 10 or 11 or 12 or 13 or 14 or 15 & 1232 \\
\hline 17 & 4 and 16 & 43 \\
\hline
\end{tabular}

(2) Database of Abstracts of Reviews of Effects from inception to 23 January 2017.

\begin{tabular}{llr}
\hline 1 & dyspepsia*.mp. & 113 \\
2 & functional dyspepsia*.mp. & 34 \\
3 & non ulcer dyspepsia*.mp. & 22 \\
4 & 1 or 2 or 3 & 113 \\
5 & Chinese herb*.mp. & 356 \\
6 & herb*.mp. & 508 \\
7 & Traditional Chinese medic*.mp. & 82 \\
8 & phytother*.mp. & 260 \\
9 & (chinese adj5 (traditional or medic*)l.mp. & 350 \\
10 & (plant or plants).mp. & 336 \\
\hline
\end{tabular}


Table A1. (Continued)

\begin{tabular}{llr}
\hline 11 & (traditional adj5 medic*l.mp. & 216 \\
12 & Chinese medic*.mp. & 150 \\
13 & oriental medic ${ }^{*} . \mathrm{mp}$. & 19 \\
14 & herbaceous agent.mp. & 0 \\
15 & medicinal plant*.mp. & 5 \\
16 & 5 or 6 or 7 or 8 or 9 or 10 or 11 or 12 or 13 or 14 or 15 & 914 \\
17 & 4 and 16 & 13 \\
\hline
\end{tabular}

(3) MEDLINE from inception to 23 January 2017 :

\begin{tabular}{|c|c|c|}
\hline 1 & search:.tw. & 305990 \\
\hline 2 & meta analysis.mp,pt. & 116082 \\
\hline 3 & review.pt. & 2320501 \\
\hline 4 & 1 or 2 or 3 & 2542882 \\
\hline 5 & exp Dyspepsia/ & 8491 \\
\hline 6 & dyspepsia*.mp. & 12156 \\
\hline 7 & functional dyspepsia*.mp. & 2311 \\
\hline 8 & non ulcer dyspepsia*.mp. & 1020 \\
\hline 9 & 5 or 6 or 7 or 8 & 12156 \\
\hline 10 & exp Drugs, Chinese Herbal/ & 41477 \\
\hline 11 & Chinese herb*.mp. & 44028 \\
\hline 12 & exp Medicine, Chinese Traditional/ & 18731 \\
\hline 13 & Traditional Chinese medic*.mp. & 13289 \\
\hline 14 & exp Phytotherapy/ & 38429 \\
\hline 15 & phytother*.mp. & 38495 \\
\hline 16 & (chinese adj5 (traditional or medic*)).mp. & 36373 \\
\hline 17 & (herbs or herbal).mp. & 63413 \\
\hline 18 & (plant or plants).mp. & 629861 \\
\hline 19 & (traditional adj5 medic*).mp. & 52240 \\
\hline 20 & 10 or 11 or 12 or 13 or 14 or 15 or 16 or 17 or 18 or 19 & 705515 \\
\hline 21 & 4 and 9 and 20 & 82 \\
\hline
\end{tabular}


Table A1. (Continued)

(4) EMABSE from inception to 23 January 2017:

\begin{tabular}{|c|c|c|}
\hline 1 & meta-analys:.mp. & 197526 \\
\hline 2 & search:.tw. & 408294 \\
\hline 3 & review.pt. & 2232214 \\
\hline 4 & 1 or 2 or 3 & 2619917 \\
\hline 5 & exp dyspepsia/ & 30355 \\
\hline 6 & dyspepsia*.mp. & 32323 \\
\hline 7 & functional dyspepsia*.mp. & 4326 \\
\hline 8 & non ulcer dyspepsia*.mp. & 1340 \\
\hline 9 & 5 or 6 or 7 or 8 & 32323 \\
\hline 10 & exp Chinese medicine/ & 36292 \\
\hline 11 & exp oriental medicine/ & 3094 \\
\hline 12 & exp herbaceous agent/ & 44067 \\
\hline 13 & exp medicinal plant/ & 208652 \\
\hline 14 & exp Chinese herb/ & 5354 \\
\hline 15 & Chinese medic*.mp. & 51163 \\
\hline 16 & oriental medic*.mp. & 3892 \\
\hline 17 & herbaceous agent.mp. & 44069 \\
\hline 18 & medicinal plant*.mp. & 88505 \\
\hline 19 & Chinese herb*.mp. & 12932 \\
\hline 20 & herb*.mp. & 148695 \\
\hline 21 & 10 or 11 or 12 or 13 or 14 or 15 or 16 or 17 or 18 or 19 or 20 & 339476 \\
\hline 22 & 4 and 9 and 21 & 431 \\
\hline 23 & Limit 22 to human & 407 \\
\hline
\end{tabular}

(5) Wan Fang Digital Journals (Chinese) from inception to 23 January 2017:

(“系统综述” OR “荟萃分析” OR “META”) AND (“中药” OR “中草药” OR “中成药” OR“中西医” OR “中医”) AND (“功能性消化不良” OR “消化不良”) yielded 32 citations.

(6) China National Knowledge Infrastructure (Chinese) from inception to 23 January 2017:

$(\mathrm{KY}=$ ‘系统综述” $\mathrm{OR} \mathrm{KY}=$ ‘荟萃分析’ $\mathrm{OR} \mathrm{KY}=$ ‘META’) $\mathrm{AND}(\mathrm{KY}=$ ‘中药’ $\mathrm{OR} \mathrm{KY}=$ ‘中草药”

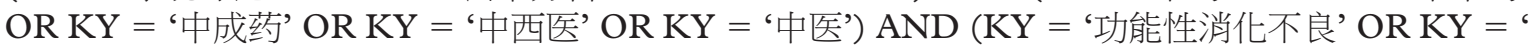
消化不良') yielded one citation.

(7) Taiwan Periodical Literature Databases (Chinese) from inception to 23 January 2017:

$(\mathrm{TX}=$ 系統綜述 $\mathrm{OR}$ 增萃分析 $\mathrm{OR}$ META $)$ [AND] $(\mathrm{TX}=$ 中藥 $\mathrm{OR}$ 中草藥 $\mathrm{OR}$ 中成藥 $\mathrm{OR}$ 中西醫 OR 中醫) [AND] $(\mathrm{TX}=$ 功能性消化不良 OR 消化不良 $)$ yielded three citations. 
(8) Chinese Biomedical Database (Chinese) from inception to 2 November 2015:

(“系统综述”[全字段] OR “荟萃分析”[全字段] OR “META”[全字段]) AND (“中药”[全字段] OR “中 草药”[全字段] OR “中成药”[全字段] OR “中西医”[全字段] OR “中医”[全字段]) AND (“功能性消化 不良”[全字段] OR “消化不良”[全字段]) yielded 20 citations.

Table A2. Detailed operational guide for applying the AMSTAR tool*.

\begin{abstract}
AMSTAR 1: Was an a priori design provided?
The research question and inclusion criteria should be established before the conduct of the review.

Operational definition (OD): a 'Yes' will be given if the review has published a protocol, ethics approval or predetermined/a priori published research objectives.
\end{abstract}

AMSTAR 2: Was there duplicate study selection and data extraction? There should be at least two independent data extractors and a consensus procedure for disagreements should be in place.

OD: a 'Yes' will be given if:(a) two reviewers performed study selection, and (b) two reviewers performed data extraction and(c) the consensus process was implemented for resolving disagreement.

AMSTAR 3: Was a comprehensive literature search performed? At least two electronic sources should be searched. The report must include years and databases used (e.g. CENTRAL, EMBASE, and MEDLINE). Key words and MESH terms must be stated and where feasible, the search strategy should be provided. All searches should be supplemented by consulting current contents, reviews, textbooks, specialized registers, or experts in the particular field of study, and by reviewing the references in the studies found.

OD: a 'Yes' will be given if at least two electronic sources plus one supplementary strategy were used le.g. Cochrane register/CENTRAL counts as two sources; a grey literature search counts as supplementary; the SIGLE database, dissertations, conference proceedings, and trial registries are all considered grey literature).

AMSTAR 4: Was the status of publication (i.e. grey literature) used as an inclusion criterion?The authors should state that they searched for reports regardless of their publication type. The authors should state whether or not they excluded any reports from the systematic review, based on their publication status, language, etc.

If review indicates that there was a search for 'grey literature' or 'unpublished literature,' indicate 'Yes.' The SIGLE database, dissertations, conference proceedings and trial registries are all considered grey for this purpose. If searching a source that contains both grey and nongrey, authors must specify that they were searching for grey/unpublished literature.

OD: if grey literature or unpublished literature was searched, a 'Yes' will be given. If eligibility criterion is restricted to 'nongrey' literature, a 'No' will be given, which indicates a methodological shortcoming in this domain.

AMSTAR 5: Was a list of studies (included and excluded) provided? A list of included and excluded studies should be provided. OD: a 'Yes' will be given if the included and excluded studies are referenced, otherwise a 'No' will be given. $\square$ Yes
$\square$ No

Yes $(a+b+c)$

Nolthese three criteria were not fulfilled)

Could not answer (i.e. not reported]

$\square$ Yes

$\square$ No

$\square$ Could not answer (i.e. not reported) $\square$ Yes

$\square$ No

Could not answer

(i.e. not reported) 
Table A2. (Continued)

AMSTAR 6: Were the characteristics of the included studies provided? In an aggregated form such as a table, data from the original studies should be provided on the participants, interventions and outcomes. The ranges of characteristics in all the studies analyzed e.g. age, race, sex, relevant socioeconomic data, disease status, duration, severity, or other diseases should be reported.

OD: a 'Yes' will be given if the information abovementioned were provided in detail, otherwise a 'No' will be given.

AMSTAR 7: Was the scientific quality of the included studies assessed and documented?

A priori methods of assessment should be provided le.g. the use of Cochrane Risk of Bias tool or Jadad scale as a mean to assessment); for other types of studies, alternative tools will also be acceptable.

OD: to score a 'Yes,' the authors should report risk of bias level in each of the methodological domain included in the risk of bias assessment tool that the authors have chosen to use.

AMSTAR 8: Was the scientific quality of the included studies used appropriately in formulating conclusions?

The results of the methodological rigor and scientific quality should be considered in the analysis and the conclusions of the review, and considerations on how risk of bias among included study may impact conclusion should be explicitly stated.

OD: to score a 'Yes,' the reviewers must consider risk of bias explicitly when writing the conclusion. For example, a reviewer may state: the results should be interpreted with caution due to high risk of bias among included studies.

AMSTAR 9: Were the methods used to combine the findings of studies appropriate?

For meta-analysis, statistical tests should be done to ensure that the studies were combinable by assessing their homogeneity. This can be done by using the Cochran $Q$ test or reporting the $I^{2}$ value.

OD: 'Yes' will be given when one of the following two situation applies:

(1) homogeneity was found, and authors used fixed effect model or random effect model, or

(2) heterogeneity was found, and authors performed appropriate subgroup analysis or meta-regression.

'No' will be given when one of the following two situations apply:

(1) heterogeneity was found, and authors used fixed or random effect model and reported the results directly without highlighting the role of heterogeneity;

heterogeneity was not assessed or reported.

AMSTAR 10: Was the likelihood of publication bias assessed? An assessment of publication bias should include a combination of graphical aids (e.g. funnel plot) or statistical tests (e.g. Egger regression test). OD: a 'No' will be given if no relevant test values or funnel plot was reported. However, a 'Yes' will still be given if authors mentioned that publication bias could not be assessed because there were fewer than 10 included studies.

AMSTAR 11: Was the conflict of interest included? Potential sources of support should be clearly acknowledged in both the systematic review and the included studies.

OD: To score a "Yes", the authors must indicate source of funding or support for the systematic review AND for each of the included studies. A "Yes" will still be scored if the authors acknowledged that funding sources for included randomized controlled trials were unknown.

*Adapted from the official AMSTAR website (http://www.amstar.ca/). 
Table A3. Details of intervention and adverse events among included systematic reviews.

\begin{tabular}{|c|c|c|c|}
\hline Author(s) & Intervention (dosage, frequency) & $\begin{array}{l}\text { Control (dosage, } \\
\text { frequency) }\end{array}$ & Adverse Events \\
\hline Liu et al. ${ }^{38}$ & $\begin{array}{l}\text { (1) Jian Pi Yi Qi formula (NR); } \\
\text { (2) Li Qi Fu Wei solution (NR); } \\
\text { (3) Jian Pi Xiao Zhang granule (NR); } \\
\text { (4) He Wei Xiao Pi capsule (NR) }\end{array}$ & $\begin{array}{l}\text { (1) Mosapride (NR); } \\
\text { (2) Domperidone (NR) }\end{array}$ & NR \\
\hline Qin et al. ${ }^{31}$ & $\begin{array}{l}\text { (1) Modified Xiao Yao San (dosage: } \\
\text { NR, q.d./ b.i.d.); } \\
\text { (2) Modified Xiao Yao San (dosage: } \\
\text { NR, b.i.d.) + } \\
\text { domperidone (10 mg, t.i.d.); } \\
\text { (3) Modified Xiao Yao San (dosage: } \\
\text { NR, b.i.d.) + } \\
\text { deanxit (1-2 tablets, t.i.d.); } \\
\text { (4) Modified Xiao Yao San (dosage: } \\
\text { NR, b.i.d.) + } \\
\text { mosabilium (5 mg, t.i.d.) }\end{array}$ & $\begin{array}{l}\text { (1) Domperidone }(10 \text { mg, } \\
\text { t.i.d.); } \\
\text { (2) Domperidone }(10 \mathrm{mg} \text {, } \\
\text { t.i.d.) + oryzanol ( } 20 \\
\text { mg, t.i.d.); } \\
\text { (3) Deanxit (1-2 tablets, } \\
\text { t.i.d.); } \\
\text { (4) Mosabilium (5 mg, } \\
\text { t.i.d.) }\end{array}$ & $\begin{array}{l}\text { Sleepiness, } \\
\text { insomnia, } \\
\text { passage of small } \\
\text { amount loose } \\
\text { stools }\end{array}$ \\
\hline Cui and Shen 29 & Details of $\mathrm{CHM}$ are not reported & $\begin{array}{l}\text { (1) Domperidone (NR); } \\
\text { (2) Mosapride (NR) }\end{array}$ & NR \\
\hline Wang et al. ${ }^{30}$ & $\begin{array}{l}\text { (1) Gan Wei Shu capsule (three } \\
\text { capsules, t.i.d., p.o.) } \\
\text { (2) Chai Hu Shu Gan powder/ } \\
\text { decoction (NR) } \\
\text { (3) He Wei Xiao Yao decoction (NR) } \\
\text { (4) Jia Wei Si Ni powder (NR) } \\
\text { (5) Xing Qi Tong Jian pill (4 Pills, } \\
\text { t.i.d., p.o.) + Ju Pi porridge (NR) } \\
\text { (6) Li Qi He Wei oral liquid (10 ml, } \\
\text { t.i.d., p.o.) } \\
\text { (7) Jiang Qi He Zhong decoction } \\
\text { (NR) } \\
\text { (8) Shu Wei decoction (NR) } \\
\text { (9) Jian Pi Shu Gan decoction (NR) } \\
\text { (10) Shu He decoction (NR) } \\
\text { (11) Modified Si Ni powder (NR) } \\
\text { (12) Shu Gan Jian Pi formula and } \\
\text { acupuncture (NR) }\end{array}$ & $\begin{array}{l}\text { (1) Domperidone }(10 \mathrm{mg} \\
\text { t.i.d.); } \\
\text { (2) Mosapride (5 mg, } \\
\text { t.i.d.); } \\
\text { (3) Polyzyme tablets } \\
\text { (three tablets, t.i.d.) }\end{array}$ & $\begin{array}{l}\text { Intervention } \\
\text { group: epigastric } \\
\text { discomfort and } \\
\text { increased passing } \\
\text { of flatus }\end{array}$ \\
\hline Xiao et al. ${ }^{32}$ & $\begin{array}{l}\text { (1) Xiang Sha Liu Jun Zi decoction } \\
\text { (dosage: NR, b.i.d.); } \\
\text { (2) Liu Jun Zi decoction (dosage: } \\
\text { NR, b.i.d.) }\end{array}$ & $\begin{array}{l}\text { (1) Domperidone (10 mg, } \\
\text { t.i.d.); } \\
\text { (2) Mosapride (5 mg, t.i.d.) }\end{array}$ & $\begin{array}{l}\text { Control group: } \\
\text { Tolerable: } \\
\text { abdominal pain, } \\
\text { diarrhea, rugitus, } \\
\text { loose stools }\end{array}$ \\
\hline Xiao et al. ${ }^{33}$ & $\begin{array}{l}\text { Shu Gan Jie Yu capsule (dosage: } \\
\text { NR, b.i.d.) }+ \\
\text { mosapride ( } 5 \text { mg, t.i.d.) }\end{array}$ & Mosapride (5 mg, t.i.d.) & $\begin{array}{l}\text { Intervention } \\
\text { group: } \\
\text { gastrointestinal } \\
\text { discomfort, dry } \\
\text { mouth, dizziness } \\
\text { Control group: dry } \\
\text { mouth, diarrhea, } \\
\text { dizziness } \\
\text { Both groups: Mild } \\
\text { and tolerable: } \\
\text { increasing } \\
\text { frequency of } \\
\text { defecation }\end{array}$ \\
\hline
\end{tabular}


Table A3. (Continued)

\begin{tabular}{|c|c|c|c|}
\hline Author(s) & Intervention (dosage, frequency) & $\begin{array}{l}\text { Control (dosage, } \\
\text { frequency) }\end{array}$ & Adverse Events \\
\hline Yang et al. ${ }^{34}$ & $\begin{array}{l}\text { (1) Modified Chai Hu Shu Gan } \\
\text { powder (dosage: NR, b.i.d.); } \\
\text { (2) Modified Chai Hu Shu Gan } \\
\text { powder (dosage: NR, b.i.d.) + } \\
\text { mosapride (5 mg, t.i.d.); } \\
\text { (3) Modified Chai Hu Shu Gan } \\
\text { powder (dosage: NR, b.i.d.) + } \\
\text { flupentixol melitracen tablets } \\
\text { (one pill, q.d.); } \\
\text { (4) Modified Chai Hu Shu Gan } \\
\text { powder (dosage: NR, b.i.d.) + } \\
\text { domperidone (10 mg, t.i.d.); } \\
\text { (5) Modified Chai Hu Shu Gan } \\
\text { powder (dosage: NR, b.i.d.) + } \\
\text { trimebutine maleate tablets } \\
\text { (100 mg, q.d.) }\end{array}$ & $\begin{array}{l}\text { (1) Domperidone (10 mg, } \\
\text { t.i.d.); } \\
\text { (2) Trimebutine maleate } \\
\text { tablets (100 mg, q.d.); } \\
\text { (3) Flupentixol melitracen } \\
\text { tablets (two pills, q.d.); } \\
\text { (4) Domperidone (10 mg, } \\
\text { t.i.d.) + } \\
\text { compound azintamide } \\
\text { Tablets (1-2 Pills, } \\
\text { t.i.d.); } \\
\text { (5) Mosapride (5 mg, t.i.d.) }\end{array}$ & $\begin{array}{l}\text { Reported as no } \\
\text { adverse events }\end{array}$ \\
\hline Gan et al. ${ }^{35}$ & Ban Xia Xie Xin decoction (NR) & $\begin{array}{l}\text { (1) Domperidone (NR) } \\
\text { (2) Mosapride (NR) }\end{array}$ & $\begin{array}{l}\text { Control group: } \\
\text { gastrointestinal } \\
\text { symptoms, } \\
\text { headache }\end{array}$ \\
\hline Fan and $\mathrm{He}^{36}$ & Da Li Tong granule (6 g, t.i.d.) & $\begin{array}{l}\text { (1) Mosapride (5 mg, } \\
\text { t.i.d.); } \\
\text { (2) Domperidone }(10 \mathrm{mg} \text {, } \\
\text { t.i.d.) }\end{array}$ & $\begin{array}{l}\text { Intervention } \\
\text { group: abdominal } \\
\text { fullness } \\
\text { Control group: } \\
\text { abdominal } \\
\text { pain, dizziness, } \\
\text { diarrhea, } \\
\text { borborygmi }\end{array}$ \\
\hline $\operatorname{Liu}^{25}$ & $\begin{array}{l}\text { (1) Xiao Pi formula no. } 1 \text { or } 2 \text { (NR) } \\
\text { (2) Li Zhong decoction (NR) } \\
\text { (3) Jian Pi Xiao Zhang powder } \\
\text { decoction (NR) } \\
\text { (4) Xiang Sha Liu Jun Zi decoction } \\
\text { (NR) } \\
\text { (5) He Wei Xiao Pi capsules (NR) } \\
\text { (6) He Wei powder (NR) } \\
\text { (7) San Jiu Wei Tai granule (NR) } \\
\text { (8) Chai Hu Shu Gan San (NR) } \\
\text { (9) Qing Song decoction (NR) } \\
\text { (10) Shu Gan Yang Xin decoction } \\
\text { (NR) } \\
\text { (11) Huo Xiang Zheng Qi capsule } \\
\text { (NR) } \\
\text { (12) Ban Xia Xie Xin decoction (NR) } \\
\text { (13) Can Ling Bai Shu San (NR) } \\
\text { (14) Zuo Jin decoction (NR) } \\
\text { (15) San Xiang decoction (NR) } \\
\text { (16) Jian Wei Xiao Zhang granule } \\
\text { (NR) } \\
\text { (17) Si Ni San (NR) } \\
\text { (18) Da Li Tong granule (NR) } \\
\text { (19) Ping Wei capsule (NR) } \\
\text { (20) Xiao Yao San (NR) } \\
\text { (21) He Wei An Xiao decoction (NR) } \\
\text { (22) Shu Gan Jie Yu capsule (NR) }\end{array}$ & $\begin{array}{l}\text { (1) Details of placebo are } \\
\text { not reported; } \\
\text { (2) Prokinetic agents/H2 } \\
\text { receptor/antagonists/ } \\
\text { antidepressants (NR) }\end{array}$ & NR \\
\hline
\end{tabular}


Table A3. (Continued)

\begin{tabular}{|c|c|c|c|}
\hline Author(s) & Intervention (dosage, frequency) & $\begin{array}{l}\text { Control (dosage, } \\
\text { frequency) }\end{array}$ & Adverse Events \\
\hline Zhang 27 & $\begin{array}{l}\text { (1) Ban Xia Xie Xin decoction (NR) } \\
\text { (2) Si Ni Xiexin decoction (NR) } \\
\text { (3) Xiao Pi Tong Jiang decoction } \\
\text { (NR) } \\
\text { (4) Chai Hu Wen Dan decoction } \\
\text { (NR) } \\
\text { (5) Zhi Shi Xiao Pi decoction (NR) } \\
\text { (6) Fu He Xie Xin decoction (NR) } \\
\text { (7) Chai Zhi Ban Xia Xie Xin } \\
\text { decoction (NR) }\end{array}$ & $\begin{array}{l}\text { (1) Domperidone (NR); } \\
\text { (2) Trimebutine (NR); } \\
\text { (3) Mosapride (NR); } \\
\text { (4) Omeprazole (NR) }\end{array}$ & NR \\
\hline Akarayosapong ${ }^{37}$ & $\begin{array}{l}\text { Liu Wei An Xiao capsule } \\
\text { (500 mg } \times 2-6 \text { pills, t.i.d.) }\end{array}$ & $\begin{array}{l}\text { (1) Mosapride (5 mg, } \\
\text { t.i.d.); } \\
\text { (2) Domperidone (10 mg, } \\
\text { t.i.d.); } \\
\text { (3) Metoclopramide (10 } \\
\text { mg, t.i.d.); } \\
\text { (4) Digestive enzymes } \\
\text { (500 mg, two pills } \\
\text { t.i.d.); } \\
\text { (5) Domperidone (10 mg, } \\
\text { t.i.d.) + oryzanol ( } 20 \\
\text { mg, t.i.d.); } \\
\text { (6) Domperidone (10 mg, } \\
\text { t.i.d.) + doxepin (12.5 } \\
\text { mg, t.i.d.); } \\
\text { (7) Flupentixol + melitracen } \\
\text { (one pill, b.i.d.); } \\
\text { (8) Domperidone (10 mg, } \\
\text { t.i.d.) + amoxicillin } \\
\text { (0.5 g, t.i.d.) }\end{array}$ & $\begin{array}{l}\text { Intervention } \\
\text { group: more } \\
\text { frequent egestion } \\
\text { Control group: } \\
\text { constipation, } \\
\text { dizziness, } \\
\text { insomnia } \\
\text { Both groups: } \\
\text { abdominal pain, } \\
\text { borborygmi, } \\
\text { more frequent } \\
\text { urination, loose } \\
\text { stool, diarrhea, } \\
\text { headache }\end{array}$ \\
\hline Ling et al. ${ }^{28}$ & Si Ni San (NR) & $\begin{array}{l}\text { (1) Domperidone (NR); } \\
\text { (2) Domperidone + } \\
\text { deanxit (NR); } \\
\text { (3) Domperidone + } \\
\text { oryzanol (NR); } \\
\text { (4) Mosapride (NR) }\end{array}$ & $\begin{array}{l}\text { Mild and tolerable } \\
\text { nausea, vomiting, } \\
\text { abdomen } \\
\text { discomfort }\end{array}$ \\
\hline Guo et al. ${ }^{26}$ & $\begin{array}{l}\text { (1) Ri Kun Shi To powder (2.5 g, } \\
\text { t.i.d.) } \\
\text { (2) Gastrosis no. } 3 \text { compound } \\
\text { granules (150 ml, b.i.d.) } \\
\text { (3) Qing Hua He Wei Tablets (four } \\
\text { tablets, } 1.2 \text { g, t.i.d.) } \\
\text { (4) Gastrosis no. } 1 \text { compound } \\
\text { granules (150 ml, b.i.d.) } \\
\text { (5) Ban Xia Xie Xin Tang granules } \\
\text { (150 ml, b.i.d.) } \\
\text { (6) Modified Liu Jun Zi Tang } \\
\text { granules (150 ml, b.i.d.) } \\
\text { (7) Nos.1-4 compound granules } \\
\text { (b.i.d./t.i.d.) } \\
\text { (8) Ban Ha Sa Si decoction (3 g, } \\
\text { t.i.d.) } \\
\text { (9) Yang Sa Yang Wei San powder } \\
\text { (9.68 g, t.i.d.) }\end{array}$ & $\begin{array}{l}\text { Placebo in the forms of } \\
\text { powder }(2.5 \mathrm{~g} / 9.68 \mathrm{~g} \text {, } \\
\text { t.i.d.), } \\
\text { granule }(150 \mathrm{~mL} / 3 \mathrm{~g} \text {, } \\
\text { b.i.d./t.i.d.) or tablets } \\
\text { (four pills, } 1.2 \mathrm{~g} \text {, t.i.d.) }\end{array}$ & NR \\
\hline
\end{tabular}

Visit SAGE journals online journals.sagepub.com/ home/tag

(SAGE journals 